\title{
Inclusive and exclusive dilepton photoproduction at high energies
}

\author{
C. Brenner Mariotto ${ }^{1}$ and M. V. T. Machado ${ }^{2}$ \\ ${ }^{1}$ Instituto de Matemática, Estatística e Física, Universidade Federal do Rio Grande, Avenue Itália, $\mathrm{km} 8$, \\ Campus Carreiros, CEP 96203-900, Rio Grande, Rio Grande do Sul, Brazil \\ ${ }^{2}$ High Energy Physics Phenomenology Group, GFPAE IF-UFRGS Caixa Postal 15051, CEP 91501-970,
} Porto Alegre, Rio Grande do Sul, Brazil

(Received 24 January 2013; published 22 March 2013)

\begin{abstract}
In this work we investigate the inclusive and exclusive photoproduction of dileptons, which is relevant for the physics programme to be studied in the proposed electron-proton collider, the LHeC. In the inclusive case, the process is sensitive to the parton distribution functions in the photon, whereas the exclusive channel is connected to the small- $x$ QCD dynamics. For the latter, we investigate the role played by saturation physics at a very high energy scenario. The estimates for production cross sections and the number of events are presented.
\end{abstract}

DOI: 10.1103/PhysRevD.87.054028

PACS numbers: 13.60.Fz, 12.40. $-\mathrm{y}, 13.60 .-\mathrm{r}, 13.90 .+\mathrm{i}$

\section{INTRODUCTION}

The parton distributions functions (PDFs) of the photon are fairly determined by current experiments. It is an open subject, the issue of generating them from available high energy data and evolving them using the perturbative QCD approach. It is timely to investigate their implications for inclusive deep inelastic processes induced by photonic parton distributions, which can be tested by the forthcoming high energy beams experiments. One of them is the deep inelastic electron-nucleon scattering at the LHC $(\mathrm{LHeC})$ machine, which is an extension of the LHC experiment as an $e p$ collider [1]. This proposed extension will open a new kinematic window-the photon-proton center-of-mass energy can reach up to $\mathrm{TeV}$ scale, which it is a very nice region for small- $x$ physics. Being specific, the energy of the incoming proton is delivered by the LHC beam, and a list of possible scenarios are considered for the energy of the incoming electron as $E_{p}=7 \mathrm{TeV}$ and $E_{e}=$ 50-200 GeV, corresponding to the center-of-mass energies of $\sqrt{s}=2 \sqrt{E_{p} E_{e}} \simeq 1.18-2.37 \mathrm{TeV}$ [2]. The planned integrated luminosity is of order $10-10^{2} \mathrm{fb}^{-1}$. Here, we shall consider inclusive dilepton (Drell-Yan) production in high energy $e p$ collisions, which are mostly sensitive the quark content of photon. It is a complementary process to the heavy-quark production in $e p$ reactions that is sensitive to the gluon content of the photon.

On the other hand, the exclusive photoproduction of lepton pairs, $\gamma N \rightarrow \gamma^{*}\left(\rightarrow \ell^{+} \ell^{-}\right)+N$ (with $N=p, A$ ) can be studied using well established high energy factorization approaches. As an example, using simple models for the elementary dipole-hadron scattering amplitude that captures the main features of the dependence on atomic number $A$, on energy, and on momentum transfer $t$, the differential cross section for exclusive dilepton photoproduction can be estimated [3]. Such an investigation is complementary to conventional partonic description of timelike Compton scattering, which considers quark handbag diagrams at leading order in $\alpha_{s}$ and simple models of the relevant generalized parton distributions (GPDs). These calculations are as well input in electromagnetic interactions in $p p$ and $A A$ collisions to be measured at the LHC. They can also be studied with beams of protons or antiprotons as recently done by the Tevatron machine. For instance, the CDF Collaboration studied the exclusive production of muon pairs, $p \bar{p} \rightarrow p \bar{p}+\mu^{+} \mu^{-}$, at lower invariant masses [4].

The aim of this work is twofold; first, we show predictions for the photoproduction of dileptons at planned $\mathrm{LHeC}$ energies within high energy factorization schemes. The inclusive photoproduction cross section including the resolved and direct contributions is calculated, focusing mostly on the dilepton invariant mass distribution. We then move to the dilepton exclusive photoproduction, analyzing the production cross section using the color dipole picture which gives us a simple picture of the process at the small- $x$ regime. The present work is complementary to the calculation presented in Ref. [5], where the photoproduction of massive gauge bosons was investigated as a tool to test the standard model and at same time to gain insight on a very specific physics beyond SM, namely, the $W W \gamma$ couplings. There, the production of $W^{ \pm}, Z$ was computed and predictions were made for the number of events produced at the $\mathrm{LHeC}$ regime. This article is organized as follows. The basic formulas to calculate the inclusive and exclusive (diffractive) photoproduction of virtual photons are presented in next section. Our numerical results for cross section and event rates are presented in Sec. III, followed by the correspondent discussion. The summary and conclusions are presented in Sec. IV.

\section{INCLUSIVE AND EXCLUSIVE DILEPTON PHOTOPRODUCTION}

Let us start by considering the Drell-Yan inclusive cross section at next-to-leading order (NLO) for the process $\gamma p \rightarrow \ell^{+} \ell^{-}+X$. In particular, we will use the NLO 
matrix elements and PDFs in the $\overline{M S}$ scheme. The differential cross section on the dilepton mass is given by [6]

$$
\begin{aligned}
\frac{d \sigma}{d M^{2}}= & \frac{4 \pi \alpha^{2}}{3 M^{2}} \int_{\tau}^{1} \frac{d x_{p}}{x_{p}} \int_{\tau / x_{p}}^{1} \frac{d x_{\gamma}}{x_{\gamma}} \\
& \times \sum_{q=u, d, s, c} e_{q}^{2}\left\{P _ { q q } ( x _ { p } , x _ { \gamma } , Q ^ { 2 } ) \left[\delta\left(1-\frac{\tau}{x_{p} x_{\gamma}}\right)\right.\right. \\
& \left.+\frac{\alpha_{s}\left(\mu^{2}\right)}{2 \pi} f_{q \bar{q}}\left(\frac{\tau}{x_{p} x_{\gamma}}\right)\right]+\frac{\alpha_{s}\left(\mu^{2}\right)}{2 \pi} f_{g q}\left(\frac{\tau}{x_{p} x_{\gamma}}\right) \\
& \times P_{g q}\left(x_{p}, x_{\gamma}, Q^{2}\right)+\frac{6 e_{q}^{2} \alpha}{2 \pi} f_{g q}\left(\frac{\tau}{x_{p} x_{\gamma}}\right) \\
& \left.\times P_{\mathrm{cp}}\left(x_{p}, x_{\gamma}, Q^{2}\right)\right\},
\end{aligned}
$$

where one has $\tau=M^{2} / s$. The quantities $x_{\gamma}$ and $x_{p}$ are the parton momentum fractions from the photon and proton, respectively. Here, $q_{\gamma}, g_{\gamma}$ are the quark and gluon distribution functions for the photon, whereas $q_{p}, g_{p}$ are the corresponding functions for the proton. The first term in equation above contains the dominating leading order quark-antiquark initial state and the associated real and virtual corrections. The second term includes the $g_{p}+q_{\gamma}$ and $q_{p}+g_{\gamma}$ initial states whereas the last term is the Compton term. In the numerical calculations we set the factorization and renormalization scales at the invariant mass of the dilepton pair, $\mu^{2}=Q^{2}=M^{2}$. The quantities $P_{i}\left(x_{p}, x_{\gamma}, Q^{2}\right)$ are written down as

$$
\begin{gathered}
P_{q q}=q_{p}\left(x_{p}, Q^{2}\right) \bar{q}_{\gamma}\left(x_{\gamma}, Q^{2}\right)+\bar{q}_{p}\left(x_{p}, Q^{2}\right) q_{\gamma}\left(x_{\gamma}, Q^{2}\right), \\
P_{g q}=g_{p}\left(x_{p}, Q^{2}\right)\left[q_{\gamma}\left(x_{\gamma}, Q^{2}\right)+\bar{q}_{\gamma}\left(x_{\gamma}, Q^{2}\right)\right] \\
+\left[q_{p}\left(x_{p}, Q^{2}\right)+\bar{q}_{p}\left(x_{p}, Q^{2}\right)\right] g_{\gamma}\left(x_{\gamma}, Q^{2}\right), \\
P_{\mathrm{cp}}=\left[q_{p}\left(x_{p}, Q^{2}\right)+\bar{q}_{p}\left(x_{p}, Q^{2}\right)\right] \delta\left(1-x_{\gamma}\right) .
\end{gathered}
$$

The calculation above can be compared to the updated calculation for the massive gauge boson photoproduction done in Ref. [5]. They include the direct and resolved photon contributions to the production rates in an analysis designed to the $\mathrm{LHeC}$ machine.

As the inclusive dilepton photoproduction is concerned, a better experimental signal can be obtained considering their exclusive production. This is particularly true if one takes high photon luminosity as typical for coherent interactions in heavy ion colliders or $e^{+} e^{-}$colliders. The high luminosity and the very large center-of-mass energy to be available at the $\mathrm{LHeC}$ machine open a window for the possibility of large rates of exclusive dileptons. In the high energy limit it is related to the timelike Compton scattering, where a virtual photon is produced in the photoproduction process. Such a process is better viewed in the target rest frame, commonly named the color dipole approach. Let us now to introduce the relevant equations in the color dipole picture for the timelike Compton scattering. In this formalism [7], the scattering process $\gamma p \rightarrow \gamma^{*} p$ is assumed to proceed in three stages: first, the incoming real photon fluctuates into a quark-antiquark pair, then the $q \bar{q}$ pair scatters elastically on the proton, and finally the $q \bar{q}$ pair recombines to form a virtual photon (which subsequently decays into lepton pairs). The amplitude for production of the exclusive virtual photon final state is given by $[8,9]$

$$
\begin{aligned}
\mathcal{A}^{\gamma p \rightarrow \gamma^{*} p}(x, Q, \Delta)= & \sum_{f} \sum_{h, \bar{h}} \int d^{2} \vec{r} \int_{0}^{1} d z \Psi_{h \bar{h}}^{*}(r, z, Q) \\
& \times \mathcal{A}_{q \bar{q}}(x, r, \Delta) \Psi_{h \bar{h}}(r, z, 0),
\end{aligned}
$$

where $\Psi_{h \bar{h}}(r, z, Q)$ denotes the amplitude for a photon to fluctuate into a quark-antiquark dipole with helicities $h$ and $\bar{h}$ and flavor $f$. The quantity $\mathcal{A}_{q \bar{q}}(x, r, \Delta)$ is the elementary amplitude for the scattering of a dipole of size $r$ on the proton, $\vec{\Delta}$ denotes the transverse momentum lost by the outgoing proton (with $t=-\Delta^{2}$ ), $x$ is the Bjorken variable, and $Q^{2}$ is the photon virtuality. Here, we are considering the photoproduction case and then only the transversely polarized overlap function contributes. For a given quark flavor $f$, when summed over the quark helicities, it gives

$$
\begin{aligned}
\left(\Psi_{\gamma^{*}}^{*} \Psi_{\gamma}\right)_{T}^{f}= & \frac{N_{c} \alpha_{\mathrm{em}} e_{f}^{2}}{2 \pi^{2}}\left\{\left[z^{2}+\bar{z}^{2}\right] \varepsilon_{1} K_{1}\left(\varepsilon_{1} r\right) \varepsilon_{2} K_{1}\left(\varepsilon_{2} r\right)\right. \\
& \left.+m_{f}^{2} K_{0}\left(\varepsilon_{1} r\right) K_{0}\left(\varepsilon_{2} r\right)\right\}
\end{aligned}
$$

where we have defined the quantities $\varepsilon_{1,2}^{2}=z \bar{z} Q_{1,2}^{2}+m_{f}^{2}$ and $\bar{z}=(1-z)$. Accordingly, the photon virtualities are $Q_{1}^{2}=0$ (incoming real photon) and $Q_{2}^{2}=-Q^{2}$ (outgoing virtual photon). For simplicity, we consider space-like kinematics. In Ref. [10], the complete timelike calculation is performed in the $k_{\perp}$ formalism and the results are somewhat larger than the presented here. Therefore, the current estimation is a lower bound for the exclusive dilepton protoproduction.

Taking the imaginary part of amplitude, which is dominant at high energies, the elastic diffractive cross section is then given by

$$
\frac{d \sigma^{\gamma p \rightarrow \gamma^{*} p}}{d t}=\frac{1}{16 \pi}\left|\mathcal{A}^{\gamma p \rightarrow \gamma^{*} p}(x, Q, \Delta)\right|^{2} .
$$

In our numerical calculations we consider saturation models which correctly describe exclusive processes at high energies like vector meson production, diffractive deep inelastic scattering and deeply virtual Compton scattering (DVCS). Our baseline model will be nonforward saturation model of Ref. [9]. Its advantage is to give the $t$ dependence of elastic differential cross section without the necessity of considerations about the impact parameter details of the process and the overall normalization is 
determined without assumptions about the elastic slope. The nonforward scattering amplitude can be written as

$$
\mathcal{A}_{q \bar{q}}(x, r, \Delta)=2 \pi R_{p}^{2} e^{-B|t|} N\left(r Q_{\text {sat }}, x\right),
$$

with the asymptotic behaviors $Q_{\text {sat }}^{2}(x, \Delta) \sim \max \left(Q_{0}^{2}, \Delta^{2}\right) \times$ $\exp [-\lambda \ln (x)]$. Specifically, the $t$ dependence of the saturation scale is parametrized as

$$
Q_{\text {sat }}^{2}(x,|t|)=Q_{0}^{2}(1+c|t|)\left(\frac{1}{x}\right)^{\lambda}
$$

in order to interpolate smoothly between the small and intermediate transfer regions. The form factor $F(\Delta)=$ $\exp (-B|t|)$ catches the transferred momentum dependence of the proton vertex, which is factorized from the projectile vertices and does not spoil the geometric scaling properties. Finally, the scaling function $N$ is obtained from the forward saturation model [11], whose functional form resembles the analytical solution for the nonlinear QCD equations in the asymptotic energy regime. Here, we investigate the exclusive photoproduction of a heavy timelike photon which decays into a lepton pair, $\gamma p \rightarrow \ell^{+} \ell^{-} p$. Therefore, for the $\ell^{+} \ell^{-}$invariant mass distribution from the virtual $\gamma^{*}$ decay, we have (with $Q^{2}=M_{\ell^{+} \ell^{-}}^{2}$ )

$\frac{d \sigma}{d M_{\ell^{+} \ell^{-}}^{2}}\left(\gamma p \rightarrow \ell^{+} \ell^{-} p\right)=\frac{\alpha_{\mathrm{em}}}{3 \pi M_{\ell^{+} \ell^{-}}^{2}} \sigma\left(\gamma p \rightarrow \gamma^{*} p\right)$.

In the next section we compute the numerical results for the inclusive and exclusive photoproduction of dileptons focusing on $\mathrm{LHeC}$ regime of energy/luminosity. We also investigate the sensitivity to the theoretical uncertainties for both processes.

\section{NUMERICAL RESULTS AND DISCUSSIONS}

Our starting point is a study of the inclusive dilepton photoproduction for the proposed LHeC machine [1,2]. Using the design with an electron beam having a laboratory energy of $E_{e}=70 \mathrm{GeV}$, the center-of-mass energy will reach $E_{\mathrm{cm}}=W_{\gamma p}=1.4 \mathrm{TeV}$ and a nominal luminosity of order $10^{33} \mathrm{~cm}^{-2} \mathrm{~s}^{-1}$. Figure 1 presents the differential cross section, Eq. (1), as a function of the dilepton invariant mass (with $M_{\ell^{+} \ell^{-}}>3 \mathrm{GeV} / \mathrm{c}$ ). Here, we have summed the resolved and direct contributions and through the calculations proton structure functions of CTEQ6M [12] and photon structure functions of GRV [13] have been used with $\mu^{2}=M_{\ell^{+} \ell^{-}}^{2}$. For sake of comparison, the result for the DESY-HERA energy range is also shown. When comparing the distinct energies, deviations are larger for higher invariant masses. The behavior is similar for small invariant mass and the overall normalization differs by a factor about ten. Our estimates for the inclusive DY photoproduction cross sections are the following. One gets $\sigma_{\text {inc }}\left(\gamma+p \rightarrow \ell^{+} \ell^{-} X\right) \simeq 0.78 \mathrm{nb}$ for the integrated cross section with $M_{\ell^{+} \ell^{-}} \geq 3 \mathrm{GeV} / \mathrm{c}$. This results can be compared to the HERA energy, which gives an integrated cross

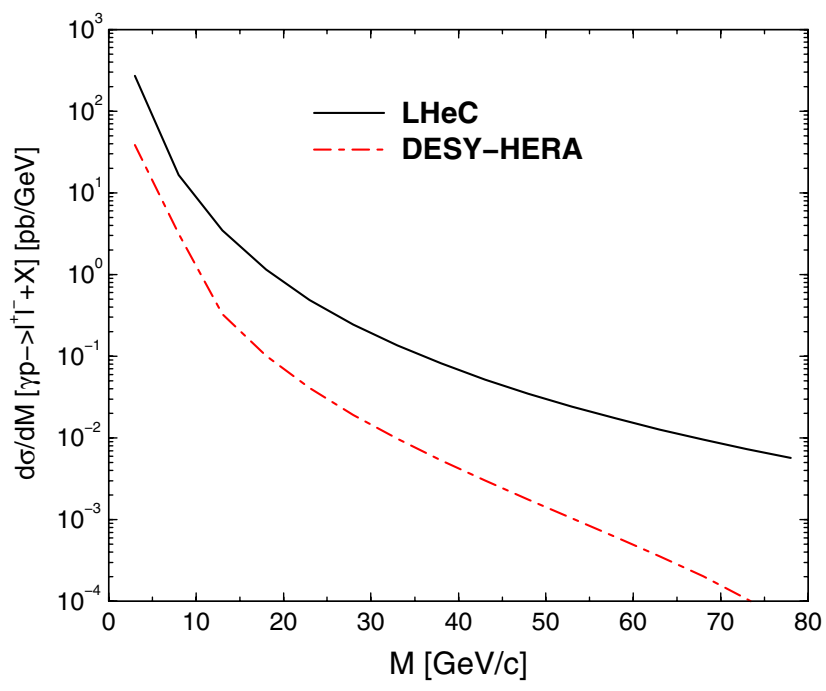

FIG. 1 (color online). Differential cross sections for the inclusive photoproduction of dileptons as a function of dilepton invariant mass for DESY-HERA and LHeC energies.

section of $0.12 \mathrm{nb}$. These are rough estimates once we have not introduced the theoretical uncertainty coming from using distinct PDFs for proton and photons. The uncertainty concerning the variation of hard scale is small in a NLO QCD computation.

We also compute the electron-proton total cross sections for mass cut $M_{\ell^{+} \ell^{-}} \geq 3 \mathrm{GeV} / \mathrm{c}$ and the corresponding number of events. The latter has been computed using $N_{\mathrm{ev}}=\sigma\left(e p \rightarrow \ell^{+} \ell^{-}+X\right) L_{\mathrm{int}}$. At this point, we consider the acceptance in the dilepton channel as $100 \%$. The photoproduction cross section is calculated by convoluting the Weizsäcker-Williams spectrum

$$
\begin{aligned}
f_{\gamma / e}(y)= & \frac{\alpha}{2 \pi}\left[\frac{1+(1-y)^{2}}{y} \log \frac{Q_{\max }^{2}}{Q_{\min }^{2}}\right. \\
& \left.-2 m_{e}^{2} y\left(\frac{1}{Q_{\min }^{2}}-\frac{1}{Q_{\max }^{2}}\right)\right],
\end{aligned}
$$

with the differential hadronic cross section. One has $Q_{\min }^{2}=m_{e}^{2} y /(1-y)$ and we impose a cut of $Q_{\min }^{2}=$ 0.01 . An integrated luminosity $L_{\text {int }}$ of $10 \mathrm{fb}^{-1}$ [2] is assumed in order to compute the number of events, $N_{\mathrm{ev}}$. The final result is $\sigma\left(e p \rightarrow \ell^{+} \ell^{-}+X\right)=11 \mathrm{pb}$ for the LHeC energy regime and the number of events is around $1.1 \times 10^{5}$. The number of events is considerable as expected for an inclusive process.

Let us now move to the exclusive dilepton photoproduction. In the following we compute the integrated cross section, performing the integration over $|t| \leq 1 \mathrm{GeV}^{2}$. It should be noticed that the $|t|$ dependence is distinct from other implementations of the dipole cross section. However, for integrated cross sections, these deviations do not play an important role. In Fig. 2 we focus on the invariant mass $\left(M_{\ell^{+} \ell^{-}}^{2} \geq 1 \mathrm{GeV}^{2} / \mathrm{c}^{2}\right)$ dependence for fixed values of energy, as computed from Eqs. (7) and (10). Once again we 


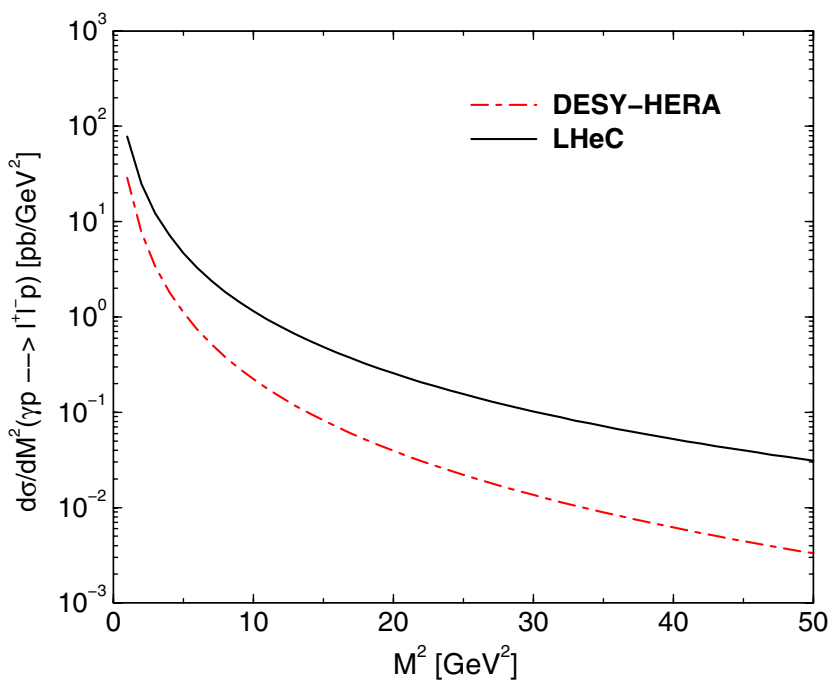

FIG. 2 (color online). Differential cross sections for the exclusive photoproduction of dileptons as a function of dilepton invariant mass for DESY-HERA and LHeC energies.

show the results for the LHeC energy and DESY-HERA for comparison. Accordingly, the spectrum is dominated by small invariant masses of order $M_{\ell^{+} \ell^{-}}^{2}<20 \mathrm{GeV}^{2}$, independent of energy. In the saturation models, the qualitative behavior can be obtained considering dominance of small size dipoles configuration. The forward amplitude reads as $\mathcal{A} \propto\left(Q_{\text {sat }}^{2} / M^{2}\right)$ times a logarithmic enhancement on $M^{2}$. Therefore, qualitatively the differential cross section behaves like $d \sigma / d M^{2} \propto\left(M^{2}\right)^{-\delta}\left[1+\log \left(M^{2}\right)\right]$, with $\delta \approx 3$. The total cross section, $\sigma\left(\gamma p \rightarrow \ell^{+} \ell^{-} p\right)$, integrated over dilepton invariant mass $M_{\ell^{+} \ell^{-}} \geq 1.5 \mathrm{GeV}$ for energies $W \gg 10^{2} \mathrm{GeV}$ can be adjusted by a power fit $\sigma=$ $3 \mathrm{pb}\left(W / W_{0}\right)^{0.46}$, with $W_{0}=1 \mathrm{GeV}$. The integrated cross section gives $\sigma_{\text {exc }}\left(\gamma+p \rightarrow \ell^{+} \ell^{-}+p\right) \simeq 80 \mathrm{pb}$ with the cut $M_{\ell^{+} \ell^{-}} \geq 1.5 \mathrm{GeV} / \mathrm{c}$ and also one has $\sigma(e p \rightarrow$ $\left.\ell^{+} \ell^{-}+p\right)=2.1 \mathrm{pb}$ for the $\mathrm{LHeC}$ energy regime and the number of events is around $2 \times 10^{4}$. The number of events is one order of magnitude smaller than for the inclusive case. This is consistent with the HERA trend of a $10 \%$ ratio for diffractive over inclusive processes. The extrapolation of current study for the electroproduction case is straightforward, as within the color dipole approach the deeply virtual Compton cross section is, in general, following the geometric scaling property. That is, $\sigma_{\text {Compton }}\left(x, Q^{2}\right)=$ $\sigma_{\text {Compton }}(\tau)$, where the scaling variable $Q$ is given in terms of incoming photon virtuality and the corresponding saturation scale, $\tau=Q^{2} / Q_{\text {sat }}^{2}(x)$.

It is timely to discuss here the limitation of the spacelike kinematics considered in current calculation. In such a case, the wave function for an incoming photon with spacelike virtuality $q^{2}=-Q^{2}<0$ is very well known and can easily be used to estimate the exclusive diffractive cross section, taking $Q^{2}=M_{\ell^{+} \ell^{-}}^{2}$ for the virtuality of final state photon. Within the color dipole approach, the correct timelike kinematics have been addressed in Ref. [14] in the scope of exclusive diffractive photoproduction of heavy gauge bosons, like $Z^{0}$ production. However, a phenomenological study for timelike Compton scattering has not been addressed yet. The main difficulty is the numerical computation of scattering amplitude as the wave function for an outgoing photon with timelike $q^{2}=M_{\ell^{+} \ell^{-}}^{2}>0$ leads to an overlap function which gives an integrand strongly oscillatory as a function of transverse dipole size $r$ as noticed in Ref. [14]. On the other hand, the calculation of timelike Compton scattering using the $k_{\perp}$-factorization formalism has been done in Ref. [10] as discussed in previous section. It was found the ratio of the cross section $R$ (timelike/spacelike) is $q^{2}$ dependent and is of order 3 or 4 at high energies.

We notice that the nonforward amplitude is assumed to be partially factorized concerning the $x$ and $t$ dependences, Eqs. (8) and (9), as the saturation scale also depends on the momentum transfer $t$. The model we are using describes correctly the $t$ dependence of vector meson electroproduction and DVCS as well. In general, within the dipole approach the extension of amplitude to finite momentum transfers is set to be completely factorized. This assumption, due to its simplicity, is widely used in the literature, although it contradicts the Regge-pole theory, as shown, e.g., in studies of DVCS or GPDs, as shown, e.g., in Refs. $[15,16]$. We believe that the distinct assumptions for the $t$ dependence will introduce an uncertainty on the overall normalization as we are considering only $t$-integrated cross sections in current study. On the other hand, this issue would be important when distributions on momentum transfer is concerned.

Finally, let us compare the present calculation to previous studies. In Ref. [6] the inclusive dilepton photoproduction has first addressed focusing on the DESY-HERA energy regime. The current calculation does an upgrade for those results using the state of art concerning new parton distribution functions for the proton and photon. Our predictions for the $\mathrm{LHeC}$ are consistent with an energy extrapolation from the HERA machine. However, the LHeC machine is probing the PDFs at smaller values of parton momentum fraction, $x_{p}$, than for HERA. For instance, for invariant mass of $4 \mathrm{GeV} / \mathrm{c}$, one has the minimum momentum fraction probed $x_{p}=8 \times 10^{-6}$ for LHeC against $x_{p}=4 \times 10^{-4}$ for HERA. A similar trend occurs for the photon parton function as $x_{p} \rightarrow 1$ and $x_{\gamma}$ goes to $M^{2} / s$. Therefore, $\mathrm{LHeC}$ will be an outstanding place to study the small- $x$ physics in addition to the PDFs for photons. Concerning the exclusive dilepton photoproduction, our estimates can be compared to recent studies in literature. For instance, in Ref. [10] the exclusive dilepton photoproduction is computed within the $k_{\perp}$-factorization formalism. We have checked that the invariant mass distribution is consistent with ours with deviations only on the overall normalization. In particular, the extrapolation for the $\mathrm{LHeC}$ energy gives an integrated cross section 
around $1 \mathrm{nb}$ (using the cut $M_{\ell^{+} \ell^{-}}^{2} \geq 1.5 \mathrm{GeV}^{2} / \mathrm{c}^{2}$ ). This is very large compared to our estimates and the origin of deviation is the fact that the correct timelike configuration produces a larger cross section compared to the spacelike configuration we have considered. This issue is discussed in detail in Ref. [10] and the main point is that the timelike calculation is a factor 3 or 4 compared to the spacelike photon configuration. In addition, the timelike Compton scattering was also investigated in Ref. [17] within the GPD factorization approach. The latter is a robust study as the interference at the amplitude level with the pure QED subprocess is also computed. For the energies we are considering here, the QED Bethe-Heitler contribution saturates around $28.5 \mathrm{pb}$ for the integrated cross section, whereas the timelike process contributes with $15-25 \mathrm{pb}$. Disregarding interference contribution (in high energies they are comparable to timelike and QED contributions), the total cross section is around $45-50 \mathrm{pb}$, which is smaller than the present calculation. The reason for a lower timelike cross section in Ref. [17] is the higher order contributions that involve gluon GPDs were not considered.

\section{SUMMARY}

We have investigated the possibility for the inclusive and exclusive dilepton detection in the photoproduction process at the proposed deep inelastic electron-nucleon scattering at the $\mathrm{LHeC}$ machine. The photon-proton cross sections have been computed for inclusive photoproduc- tion at NLO accuracy and are of order the unit of picobarns. The number of events is evaluated to be of order $10^{5}$ assuming an integrated luminosity of $10 \mathrm{fb}^{-1}$, which means that such a measurement is fairly feasible. It was shown that process is a good place to investigate constraints to the parton distribution functions for the proton and the photon at very small- $x$ region. We have investigated also the exclusive dilepton photoproduction using the $\mathrm{LHeC}$ design. It was found that the spectrum is dominated by small invariant masses and the forward amplitude scales with $\left(Q_{\text {sat }}^{2} / M^{2}\right)$ modulo logarithmic enhancements, where $Q_{\text {sat }}$ is the phenomenological parton saturation scale. Thus, the differential cross section reads as $d \sigma / d M^{2} \propto\left(M^{2}\right)^{-3}\left[1+\log \left(M^{2}\right)\right]$. The integrated cross section gives $\sigma_{\text {exc }}\left(\gamma+p \rightarrow \ell^{+} \ell^{-}+p\right) \simeq 80 \mathrm{pb}$ with the cut $M_{\ell^{+} \ell^{-}} \geq 1.5 \mathrm{GeV} / \mathrm{c}$ and also one has $\sigma(e p \rightarrow$ $\left.\ell^{+} \ell^{-}+p\right)=2.1 \mathrm{pb}$ for the LHeC energy regime and the number of events is around $1.2 \times 10^{4}$. It can be considered a lower bound for predictions as the timelike photon configuration would give cross sections bigger by a factor of three or four. The number of events is one order of magnitude smaller than for the inclusive case, whereas the experimental detection is improved due to the presence of one rapidity gap in final state.

\section{ACKNOWLEDGMENTS}

This research was supported by CNPq and FAPERGS, Brazil.
[1] J. B. Dainton, M. Klein, P. Newman, E. Perez, and F. Willeke, J. Instrum. 1, P10001 (2006).

[2] J.L.A. Fernandez et al. (LHeC Study Group Collaboration), J. Phys. G 39, 075001 (2012).

[3] M. V. T. Machado, Phys. Rev. D 78, 034016 (2008).

[4] A. Aaltonen et al. (CDF Collaboration), Phys. Rev. Lett. 102, 242001 (2009).

[5] C. B. Mariotto and M. V. T. Machado, Phys. Rev. D 86, 033009 (2012).

[6] A. C. Bawa, K. Charchula, and W. J. Stirling, Phys. Lett. B 313, 461 (1993).

[7] N. N. Nikolaev and B. G. Zakharov, Z. Phys. C 49, 607 (1991); 53, 331 (1992); A. H. Mueller, Nucl. Phys. B415, 373 (1994); A. H. Mueller and B. Patel, Nucl. Phys. B425, 471 (1994).

[8] H. Kowalski, L. Motyka, and G. Watt, Phys. Rev. D 74, 074016 (2006).
[9] C. Marquet, R. Peschanski, and G. Soyez, Phys. Rev. D 76, 034011 (2007).

[10] W. Schäfer, G. Ślipek, and A. Szczurek, Phys. Lett. B 688 , 185 (2010).

[11] E. Iancu, K. Itakura, and S. Munier, Phys. Lett. B 590, 199 (2004).

[12] J. Pumplin, D. R. Stump, J. Huston, H. L. Lai, P. Nadolsky, and W. K. Tung, J. High Energy Phys. 07 (2002) 012.

[13] M. Glück, E. Reya, and A. Vogt, Phys. Rev. D 45, 3986 (1992).

[14] L. Motyka and G. Watt, Phys. Rev. D 78, 014023 (2008).

[15] M. Capua, S. Fazio, R. Fiore, L. Jenkovszky, and F. Paccanoni, Phys. Lett. B 645, 161 (2007).

[16] L. L. Jenkovszky, Phys. Rev. D 74, 114026 (2006).

[17] B. Pire, L. Szymanowski, and J. Wagner, Phys. Rev. D 79, 014010 (2009). 